\title{
The Role of SreA-Mediated Iron Regulation in Maintaining Epichloë festucae-Lolium perenne Symbioses
}

\author{
Natasha T. Forester, ${ }^{1}$ Geoffrey A. Lane, ${ }^{1}$ Catherine M. McKenzie, ${ }^{1}$ lain L. Lamont, ${ }^{2}$ and \\ Linda J. Johnson ${ }^{1+}$ \\ ${ }^{1}$ AgResearch Ltd., Grasslands Research Centre, Tennent Drive, Private Bag 11008, Palmerston North 4442, New Zealand \\ ${ }^{2}$ Department of Biochemistry, University of Otago, P.O. Box 56, Dunedin 9054, New Zealand
}

Accepted 16 May 2019.

In ascomycetes and basidiomycetes, iron-responsive GATAtype transcriptional repressors are involved in regulating iron homeostasis, notably to prevent iron toxicity through control of iron uptake. To date, it has been unknown whether this iron regulator contributes toward mutualistic endosymbiosis of microbes with plants, a system where the endophyte must function within the constraints of an in-host existence, including a dependency on the host for nutrient acquisition. Functional characterization of one such protein, SreA from Epichlö̈ festucae, a fungal endosymbiont of cool-season grasses, indicates that regulation of iron homeostasis processes is important for symbiotic maintenance. The deletion of the sreA gene $(\Delta s r e A)$ led to iron-dependent aberrant hyphal growth and the gradual loss of endophyte hyphae from perennial ryegrass. SreA negatively regulates the siderophore biosynthesis and high-affinity iron uptake systems of E. festucae, similar to other fungi, resulting in iron accumulation in mutants. Our evidence suggests that SreA is involved in the processes that moderate Epichlo $\ddot{e}$ iron acquisition from the plant apoplast, because overharvesting of iron in $\Delta s r e A$ mutants was detected as premature chlorosis of the host using a hydroponic plant growth assay. $E$. festucae appears to have a tightly regulated iron management system, involving SreA that balances endophyte growth with its survival and prevents overcompetition with the host for iron in the intercellular niche, thus promoting mutualistic associations. Mutations that interfere with Epichlo $\ddot{e}$ iron management negatively affect iron-dependent fungal growth and destabilize mutualistic Epichlö -ryegrass associations.

Keywords: endophytes, Epichlö̈, fungus-plant interactions, GATA factor, iron homeostasis, symbiosis

The acquisition, utilization, and storage processes relating to the essential trace element iron are strictly regulated to

${ }^{\dagger}$ Corresponding author: L. J. Johnson; linda.johnson@agresearch.co.nz

Funding: This research was facilitated and supported by AgResearch Ltd. under Ministry of Business, Innovation and Employment contract A20067 from the Strategic Science Investment Fund (SSIF), New Zealand.

*The $\boldsymbol{e}$-Xtra logo stands for "electronic extra" and indicates that 10 supplementary figures and four supplementary tables are published online.

The author(s) declare no conflict of interest.

() 2019 The American Phytopathological Society maintain iron homeostasis in fungi, ensuring appropriate availability of iron for metabolism while avoiding detrimental stresses associated with iron concentration extremes. The specialized cellular systems that are employed to acquire iron from iron-limiting environments include high-affinity ferric $\left(\mathrm{Fe}^{3+}\right)$ iron-chelating compounds and membrane-bound reductive iron assimilation (RIA) systems (Stearman et al. 1996). To mitigate iron toxicity during iron excess, fungi sequester and store iron intracellularly as either ferric-chelates that are compartmentalized in vacuoles or $\mathrm{Fe}^{3+}$-siderophores (Haas et al. 2008; Li et al. 2001; Raguzzi et al. 1988). Siderophores are low molecular weight molecules with an extreme binding affinity for ferric ions (Neilands 1993). Fungi employ siderophores in extracellular iron procurement, intracellular iron sequestration and storage, and as well as intercellular iron transporters (Haas et al. 2008; Johnson 2008).

The expression of key genes involved in iron homeostatic processes is coordinated, in part, by a well-studied family of orthologous iron-responsive fungal GATA transcription factors (IRGF), including SreA (Aspergillus nidulans and A. fumigatus), Sre (Neurospora crassa), SreP (Penicillium chrysogenum), Urbs1 (Ustilago maydis), Sfu1 (Candida albicans), Cir1 (Cryptococcus neoformans), and Fep1(Schizosaccharomyces pombe) (Chen et al. 2011; Haas et al. 1997, 1999; Hwang et al. 2011; Jung et al. 2006; Oberegger et al. 2001; Pelletier et al. 2002; Schrettl et al. 2008; Voisard et al. 1993; Zhou et al. 1998). IRGFs bind to G-A-T-A DNA motifs in the promoters of target genes and exert repressory effects (Pouliot et al. 2010). Target genes include those encoding components of RIA and siderophore biosynthesis systems, siderophore transporters, antioxidants, and also other genes not intuitively connected with iron metabolism (An et al. 1997a; Hwang et al. 2011; Miele et al. 2007; Mercier and Labbé 2010; Oberegger et al. 2002; Schrettl et al. 2008). The fungal IRGF protein structure is typified by the presence of two N-terminally located zinc-finger DNA-binding motifs (ZF1 and ZF2) that each flank a conserved cysteine-rich region (CRR) known to bind as a dimer to Fe-S clusters and to interact with other Fe-S proteins (An et al. 1997b; Haas 2003; Harrison and Marzluf 2002; Kim et al. 2016; Pelletier et al. 2005). Variations in the regulatory outcomes of each IRGF exist in different fungal systems (Gauthier et al. 2010; Jung et al. 2006). IRGF deficiency in filamentous fungi is associated with partial derepression of siderophore biosynthesis during iron-replete conditions (Haas et al. 1999; Hwang et al. 2011; Oberegger et al. 2001; Zhou et al. 1998). In the presence of iron, A. fumigatus $\triangle$ sreA mutants display growth defects, accumulate iron, and are more susceptible to oxidative stress (Schrettl et al. 2008). The impact of loss of SreA on 
endophytic fungi and their symbiotic host partners, on whom they are dependent for nutrients, including iron, is completely unknown.

Epichlö (Ascomycota: Clavicipitaceae) is a genus of fungal endophytes that occupy intercellular regions within the grass shoots of members of the subfamily Pooideae (Hinton and Bacon 1985; Leuchtmann et al. 2014; White et al. 1993). The symbioses formed can range from antagonistic to mutualistic and fungal transmission of asexual species is predominantly via seed tissues (Majewska-Sawka and Nakashima 2004; Schardl 1996; Schardl et al. 2004). These noninvasive hyphae are tethered to, and their growth is inextricably coordinated with that of, their neighboring host cells and, when growth is complete, the cells remain metabolically active (Christensen et al. 1997, 2008). Furthermore, Epichlö̈ spp. are of agronomic value owing to their ability to confer to their host resistance to a wide range of biotic and abiotic stresses, via the strain-specific production and delivery of a variety of protective metabolites (Blankenship et al. 2001; Bush et al. 1997; Fleetwood et al. 2007; Johnson et al. 2013a; Spiering et al. 2005; Tanaka et al. 2005; Young et al. 2006).

Previously, we demonstrated iron-dependent functional roles for the Epichloe festucae siderophores ferricrocin (FC) and epichloënin (EA), whose production was promoted and repressed, respectively, in response to increasing iron supply (Forester et al. 2018; Johnson et al. 2013b). In this study, we have identified, isolated, and functionally characterized the E. festucae counterpart IRGF, SreA, and have investigated its role in siderophore biosynthesis and iron uptake. Additionally, we have evaluated the consequence of SreA loss to the symbiotic interaction of E. festucae with the host plant, perennial ryegrass.

\section{RESULTS}

\section{SreA is an iron responsive GATA factor.}

The IRGF SreA was identified in E. festucae strain Fl1 through tBLASTn homology searches of the E. festucae Fl1 genome project database (Schardl et al. 2013) using, as queries, the amino acid sequence of characterized IRGFs from A. nidulans (SreA, AAD25328), S. pombe (Fep1, NP592936), and $U$. maydis (URBS1, AAB05617) (Haas et al. 1999; Pelletier et al. 2002; Voisard et al. 1993). Each returned a top hit to the E. festucae M3 predicted gene model ID EfM3.021150. The predicted SreA coding sequence (CDS) was approximately $1.9 \mathrm{~kb}$ within an approximately $3.2-\mathrm{kb}$ transcript extending 0.75 and $0.6 \mathrm{~kb}$ upstream and downstream, respectively, of the CDS to produce a 571-amino-acid SreA protein sequence. The deduced amino acid sequence was compared with characterized SreA orthologs from other fungi (Supplementary Fig. S1). The alignment showed that E. festucae SreA contained features that were characteristic of GATA zinc-finger transcription factors, including two zincfinger motifs $\left(\mathrm{CX}_{2} \mathrm{CX}_{17} \mathrm{CX}_{2} \mathrm{C}\right)$ that each flank a CRR $\left(\mathrm{CX}_{5} \mathrm{CX}_{8} \mathrm{CX}_{2} \mathrm{C}\right)$, all followed by a basic domain and also a $\mathrm{C}$ terminally located coiled-coil domain that appears to be conserved in the filamentous fungi (Haas et al. 1999). Over the whole sequence, E. festucae SreA was most closely related to orthologs from another sordariomycete, $N$. crassa (Sre, 44\%), followed by the class Eurotiomycetes ( 31 to $33 \%$ ), and other species of Ascomycota (21 to $24 \%$ ) and basidiomycete fungi (14 to 16\%). These results are consistent with the identification of an orthologous SreA protein in the genome of E. festucae.

\section{SreA mutants have increased sensitivity to iron.}

To gain insights into the functional roles of E. festucae SreA, the sreA gene of wild-type (WT) strain Fl1 was deleted by targeted insertional gene replacement, and four $\Delta s r e A$ mutant colonies were examined in this study. The mutants showed consistent responses in all tests, and one mutant ( $\triangle s r e A 1-9)$ was genetically complemented (i.e., $\Delta s r e A /$ sreA strains). The in vitro growth response of colonies of $\triangle s r e A$ to iron availability was tested using defined medium (DM) (DM without added iron $[+0 \mathrm{Fe}]=\mathrm{DM} 0)$ supplemented with increasing concentrations of ferric iron (e.g., $+\mathrm{XFe}$, where $\mathrm{X}$ is the micromolar concentration of $\left.\mathrm{FeCl}_{3}\right)$. To induce iron starvation $(-\mathrm{Fe})$, the cellimpermeable ferrous iron chelator bathophenanthroline disulfonic acid (BPS) $(100 \mu \mathrm{M})$ was employed. BPS blocks iron uptake via the two component FetC/FtrA RIA system, which appears to be one of the major iron acquisition routes in E. festucae (Johnson et al. 2013b). Under iron-starved conditions $(-\mathrm{Fe}), \Delta s r e A$ produced white filamentous umbonate colonies similar to those of WT colonies (Fig. 1A). In contrast, in the presence of increasing iron supply, the $\Delta s r e A$ colony diameter became progressively smaller and raised, with a velveteen pulvinate appearance, and an orange-brown pigment developed on the underside of the colonies, with most intense coloration in the colony center (Supplementary Fig. S2). Radial growth diameter comparisons of the $\Delta s r e A$ with control strain colonies ranged from approximately 70 to $40 \%$ with increasing iron supply (Fig. 1). These results indicate that $\Delta$ sreA cultures are more sensitive to iron than WT and accumulate pigmented compounds in the mycelium in response to iron availability.

\section{SreA loss deregulates $E$. festucae siderophore production and RIA systems.}

SreA deficiency in filamentous fungi is associated with partial derepression of siderophore biosynthesis (Haas et al. 1999; Hwang et al. 2011; Oberegger et al. 2001; Zhou et al. 1998). Previously, we demonstrated in WT strains that FC and EA production was promoted and repressed, respectively, in response to increasing iron supply (Forester et al. 2018). Considering these facts, we predicted that mutation of SreA $(\Delta s r e A)$ in E. festucae would lead to derepression of iron uptake, manifesting as the accumulation of the extracellular siderophore EA, increased RIA activity, and, consequently, iron-promoted FC production. Liquid chromatography-mass spectrometry (LC-MS) analyses were performed, monitoring levels of total FC and EA (as ferriepichloenin A [FEA]) in mycelial and supernatant extracts. These extracts were prepared from E. festucae cultures (WT, $\Delta s r e A$, and $\Delta s r e A /$ sreA strains) (see Materials and Methods) grown under both low $(-\mathrm{Fe})$ and high $(+20 \mathrm{Fe})$ iron availabilities. As expected, in contrast to the WT and $\triangle s r e A / s r e A$ strains, the results indicated that the $\triangle$ sreA mutants hyperaccumulated both FC and FEA in the mycelia (Fig. 2) (the complete dataset is available in Supplementary Table S1). However, mycelial FC was increased under iron-starved as well as iron-replete conditions (approximately 15 to 24 times that of WT levels), whereas mycelial FEA levels were raised only in the presence of iron (approximately 9 times that of WT levels). Supernatants extracted from cultures of all strains displayed maximum FEA values during iron deprivation. The addition of iron led to a sharp decline in FEA in all strains to nondetectable levels, with the exception of FEA in supernatants derived from $\Delta s r e A$, which were significantly reduced but still quantifiable. These results suggest increased siderophore production and secretion in the mutant strain, possibly with delayed siderophore internalization or siderophore degradation. Furthermore, under trace iron (DM0) conditions, FC was detected in extracellular $\triangle s r e A$ extracts but not those of the WT, which suggest that the $\Delta s r e A$ cells are leaky.

Given the observed conservation of SreA repressor function on siderophore biosynthesis in E. festucae, we additionally 
investigated iron-dependent production of transcripts encoding FetC, the ferroxidase of the RIA membrane system whose activity is derepressed by sreA gene deletion in A. fumigatus (Schrettl et al. 2008). WT, $\Delta s r e A$, and $\Delta s r e A /$ sreA strains were grown under iron-starved (100 $\mu \mathrm{M}$ BPS) and iron-sufficient $(20 \mu \mathrm{M})$ conditions. Real-time quantitative PCR (qRT-PCR) (Fig. 3) of $f e t C$ transcripts in iron-starved (-Fe) relative to ironsupplied $(+\mathrm{Fe})$ conditions indicated that the relative number of

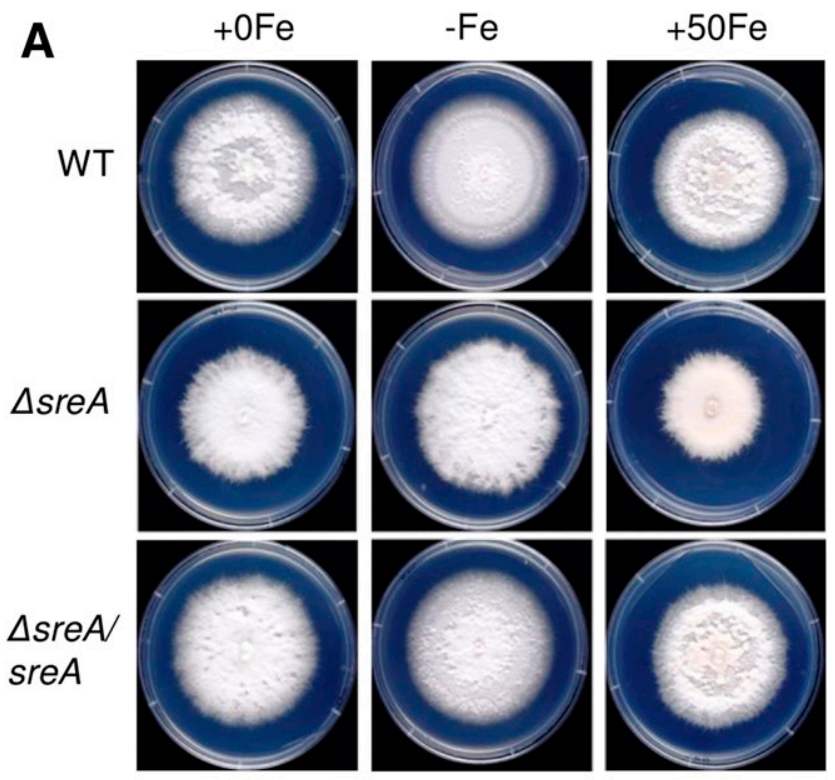

B

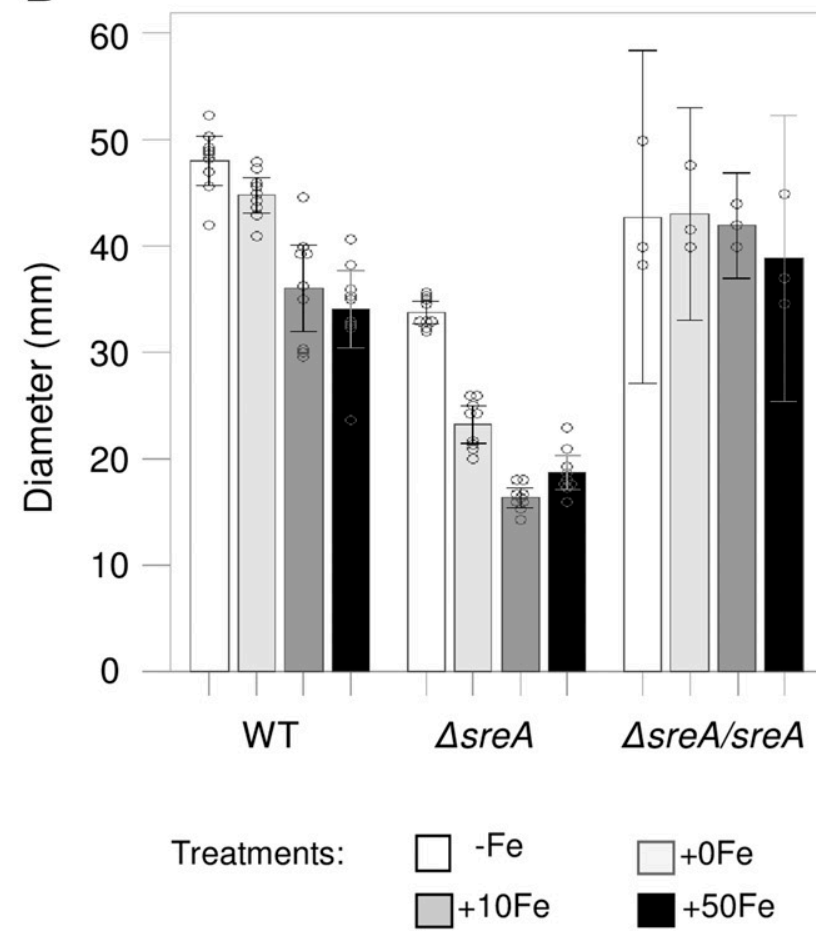

Fig. 1. Effect of iron on Epichloëfestucae growth. A, Aerial view of colony morphology of 30-day-old wild-type (WT), $\Delta s r e A$, and $\Delta s r e A /$ sreA colonies grown on defined medium without added iron (DM0), with variable iron supplementation; that is, iron-deprived $(-\mathrm{Fe})$, or with the indicated micromolar concentration of $\mathrm{FeCl}_{3}(+\mathrm{Fe})$. $\mathbf{B}$, Radial growth measurements (in millimeters) of the colonies represented in $\mathrm{A}$ at 21 days of incubation. The treatment mean (bar height) was calculated as the average of the average of measurements obtained from three colonies per plate (open circles) in triplicate for each strain (three independent transformants per strain). Error bars are $95 \%$ confidence interval (Bonferri; Minitab). transcripts was raised in all strains and not significantly different from one to another. This is consistent with the role of RIA in iron uptake during iron-limiting conditions. In contrast to the control strains (WT and $\Delta s r e A / s r e A$ ), in the iron-fed $(+\mathrm{Fe})$ cultures whose $\mathrm{fet} C$ transcripts were reduced by iron addition, those of the $\Delta s r e A$ strains remained elevated $(2.6 \pm 0.7$ fold change, $P=0.037$ by one-way analysis of variance [ANOVA]), indicating loss of the repressor function of SreA. These results confirm a central role for SreA in regulating siderophore biosynthesis and iron uptake activities under ironreplete growth conditions.

\section{SreA mutants exhibit atypical in planta growth} but do not alter host phenotype of soil-grown plants.

To investigate the effect of SreA deletion on the E. festucaeLolium perenne symbiosis, ryegrass seedlings were inoculated with WT, $\Delta s r e A$, or $\Delta s r e A / s r e A$ cultures and the symbiota were assessed for phenotypic changes. Asymptomatic systemic $\Delta s r e A$ infections of $>70$ independent hosts were established in the study. We did not detect any change in host plant development compared with control plants infected with either WT or $\Delta s r e A /$ sreA strains as determined by visual examination of infected plants' architecture and coloration (Supplementary Fig. S3). However, microscopic examination of in planta hyphae (10 to 12 weeks postinfection) using aniline blue as the contrast dye revealed that, unlike the synchronized intercellular growth observed for WTinfected plants ( 1 to 2 hyphae per apoplasmic channel), $\Delta s r e A$ hyphae were often malformed and formed clusters of hyphal networks that were unusually associated with fully expanded plant cells (Fig. 4A and B). Similar hyphal networks were observed at the base of the leaf sheath of plants infected with the control strains but seldom among expanded plant cells. The aniline blue staining of $\Delta s r e A$ hyphae was denser than for the WT and there was substantially more debris in the specimen preparation, suggesting that the structures are easily damaged (Supplementary Fig. S4). The occurrence of these networks was so abundant that their development could be followed (Supplementary Fig. S5). The structures appear to arise as a result of repetitions of lateral outgrowths or branches, seemingly from random positions along their originating hyphal compartments (individual units or cells of a hypha that are separated by internal walls or septa). Abnormal branch junctions and fused mycelial clusters were additionally observed in the $\Delta s r e A$-infected but not in WT-infected plants (Fig. 4A and B). The compartment lengths of the most regular-appearing $\Delta s r e A$ hyphal strands were measured from multiple confocal image captures ( $>10$ fields of view) for at least three symbiota derived from at least three independent $\Delta s r e A$ transformants (Fig. 4B). The $\Delta s r e A$ hyphal compartments were significantly shorter $(P=0$, ANOVA); that is, averaging $60 \%(18.5 \pm 0.84 \mu \mathrm{m})$ that of the WT $(30.8 \pm 2.6 \mu \mathrm{m})$ and $69 \%$ that of the complemented strain $(26.9 \pm 2.0 \mu \mathrm{m})$. Additionally, transmission electron microscopy of WT and $\Delta s r e A$ hyphae was employed to examine the contents of transverse sections. Hyphae were observed sporadically in vascular bundles in WT and $\Delta s r e A /$ sre $A$ hyphae but almost never in the $\Delta s r e A$ mutants (not shown). The hyphal diameters of WT, $\Delta s r e A$, and $\Delta s r e A /$ sreA were measured and those of $\triangle s r e A$ were found to be, on average, significantly larger $(2.0 \pm 0.32 \mu \mathrm{m})$ than those of the control strains (WT, $1.5 \pm 0.1$ and $\Delta s r e A / s r e A, 1.6 \pm 0.22 ; P=0.004$, ANOVA) (Fig. 4C and D). However, intracellular defects were not detected when compared with the WT. These results indicate that $\Delta s r e A$ hyphae are shorter and larger than WT or $\Delta s r e A /$ sreA hyphae.

\section{Reduced persistence of $\Delta s r e A$ in host plants.}

Although $\Delta s r e A$ fungi established systemic infections as confirmed by tissue print immunoblot tests (TPIB), subsequent 
TPIB tests performed to monitor the proportion of remaining infected plants indicated a higher proportion of loss of $\Delta s r e A$ hyphae from their hosts than of WT hyphae. For example, only $40 \%$ (7 of 17 ) of $\Delta s r e A$ hosts compared with $89 \%$ (16 of 18) of WT host plants remained infected at approximately 1.5 to 2 years postinoculation (approximately 2012 to 2014). Six months later, $80 \%$ (12 of 15) were still infected with the WT, whereas six of the seven previously $\Delta s r e A$-infected plants were uninfected, suggesting an issue with $\Delta s r e A$ transmission in vegetative host tissue.

To follow this observation further, more recently formed symbiota (aged 1.5 to 2 years) that had been verified at December 2016 as infected with either WT-FL1, $\Delta s r e A$, or $\Delta$ sreA/sreA ( $n=23,21$, and 15 infected plant genotypes, respectively) were subjected to TPIB testing. In mid-2018, 10 tillers per plant were blotted and the identity of the resident endophyte was determined using PCR.

DNA banding patterns produced from a series of PCRs from genomic DNA (gDNA), using multiple primer pairs, were diagnostic for each DNA source (e.g., from WT, mutant, fosmid, or split marker constructs). The identities of the WT and $\Delta s r e A$ strains were confirmed by these PCRs but, for the $\Delta s r e A /$ sreA strains, only 4 of 18 infected plants (representing 15 symbioses, 3 of them with 2 independently grown symbiotic clonal plants) displayed the expected pattern (presence of a 191-bp PCR product) for the complemented strain. The remainder gave variant banding patterns suggestive of DNA rearrangements occurring within the complemented strains (Supplementary Fig. S6). Significantly, a 248-bp PCR product, derived from the complement-generating fosmid, was amplified in plants that had been infected with $\Delta s r e A / s r e A$, confirming inoculation with the correct strain. Furthermore, for all three sets of clonally propagated plants, one plant retained a complemented strain while the other contained a variant version of the complemented strain. The results suggest that the in planta $\Delta s r e A /$ sreA strains are exhibiting genetic instability, probably the result of DNA rearrangements between $\triangle s r e A$ gDNA and the fosmid-derived DNA.

The resulting average percentages of tillers infected with verified endophyte were $77 \pm 31 \%(n=23)$ for the WT, $51 \pm$ $33 \%(n=21)$ for $\Delta s r e A$, and $80 \pm 27 \%(n=4)$ for $\Delta s r e A /$ sreA (Fig. 5), indicating reduced infection in $\triangle s r e A$-infected plants. A one-way ANOVA indicated a significant difference between strains $(P=0.021)$, with values for plants infected with the WT and $\triangle s r e A$ grouping separately according to Tukey's pairwise comparison. However, there were likely insufficient plants for the statistical analysis of the $\Delta$ sreA/sreA-infected plants, which grouped with both strains (albeit with values trending toward the WT). Collectively, the results indicate that the $\Delta s r e A$-grass symbiosis is not systemically maintained in the long term.

\section{$\Delta s r e A$ may overharvest host iron.}

To explore any iron-dependent effects of the $\Delta s r e A$ mutation on the symbiotic association, tillers infected with WT, $\Delta s r e A$, or $\triangle$ sreA/sreA hyphae were subjected at least three times to hydroponic growth using a range of iron concentrations ( 0 to $50 \mu \mathrm{M})$. During the course of 20 -day experiments, we observed premature chlorosis in the youngest leaves of plants infected with $\Delta s r e A$ (relative to the WT) when grown under lower iron
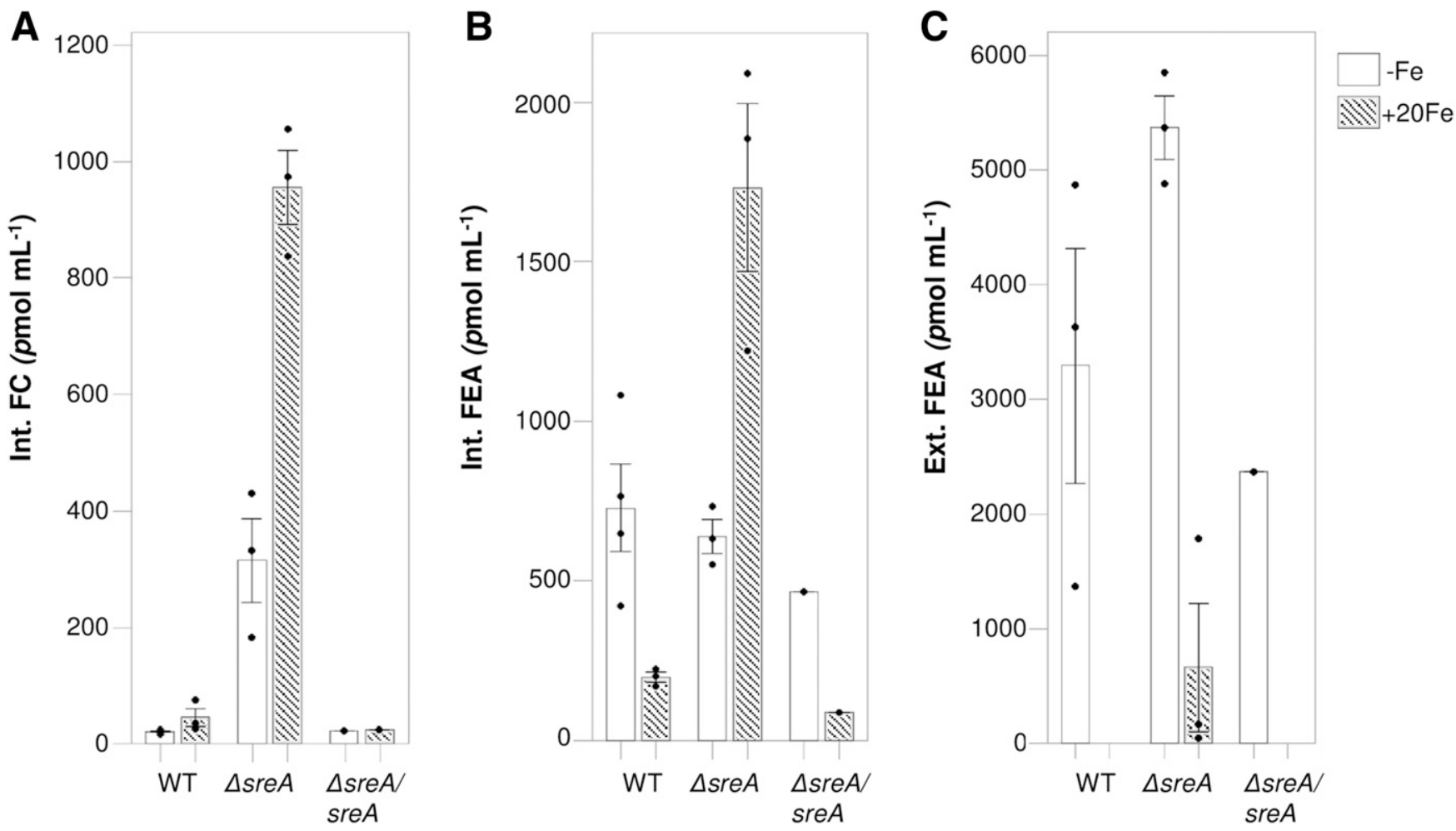

Fig. 2. Siderophore production in Epichloë festucae $\Delta$ sreA mutants. Quantification of A, intracellular ferricrocin (FC) and $\mathbf{B}$, ferriepichloenin A (FEA) in $\mathbf{C}$, mycelia and extracellular FEA from culture supernatant extracts of wild-type (WT) E. festucae and $\Delta$ sreA mutants under iron-depleted or iron-replete growth conditions. Values are reported in picomoles per milliliter of fungal culture and were obtained as previously described by Forester et al. (2018). Starter cultures of strains were mixed 1:1 in defined medium without added iron (DM0) containing appropriate additives to give the following concentrations: DM0 + $100 \mu \mathrm{M}$ bathophenanthroline disulfonic acid (-Fe) or $20 \mu \mathrm{M} \mathrm{FeCl}_{3}(+20 \mathrm{Fe})$ and grown at $23^{\circ} \mathrm{C}$ for 5 days prior to culture harvest. Limit of detection (LOD) and limit of quantification (LOQ) maxima were 5.6 and $9.3 \mathrm{pmol} \mathrm{m}^{-1}$ of culture for FC and 36 and $75 \mathrm{pmol} \mathrm{ml}^{-1}$ of culture for FEA, respectively. Dots denote data points of biological replicates for the WT $(n=4), \Delta s r e A(n=3)$, and $\Delta s r e A / s r e A(n=1)$, each strain regenerated from individual protoplasts. Error bars are standard error of the mean. 
availabilities (i.e., 0.2 to $0.5 \mu \mathrm{M}$ ) but not with iron concentrations greater than $10 \mu \mathrm{M}$ or under iron deprivation (not shown). Despite fully complementing in vitro phenotypes, the $\Delta$ sreA/sreA-infected plants showed variable complementation of the $\triangle s r e A$ phenotype under these conditions and were not included in subsequent investigations. Later, with a separate set of plants infected with $\Delta s r e A$-complemented strains, we found evidence of genetic instability (above). To reproduce the effect of low iron supply on $\Delta s r e A$-infected plants, the experiment was replicated in two randomized adjacent blocks (Supplementary Fig. S7). In all, 30 independent symbioses of ryegrass infected with the WT and $\Delta s r e A$ ( 15 plant genotypes each) were phenotyped under three iron concentrations $(0.125,0.25$, and $0.5 \mu \mathrm{M}$ ferric iron delivered as EDTA ferric sodium salt in hydroponic solution). Chlorophyll measurements were recorded for the youngest (L1) and second-youngest (L2) leaf at time points over 20 days (Materials and Methods). The results (Fig. 6; Supplementary Table S2) corroborated earlier observations that there was an effect on total chlorophyll (TC) production on L1 and L2 (strain-day effect, $P<0.0001$ and 0.029 , respectively) caused by the $\Delta s r e A$ strain in combination with low iron supply. The chlorophyll readings became significantly different, for $\Delta s r e A$ - relative to WT-infected plants, from day 9 for L1 and for L2, with this difference increasing with time (Fig. 6). The iron concentrations had no effect on the mean TC measurements of L1 (iron concentration, $P=0.3125$; strainiron concentration, $P=0.334$; strain-iron concentration -day, $P=0.669)$. However, there was a significant effect $(P<0.001)$ on L2 TC levels, with the $0.125-\mu \mathrm{M}$ iron treatment subjects showing a delayed decline in TC compared with the other iron

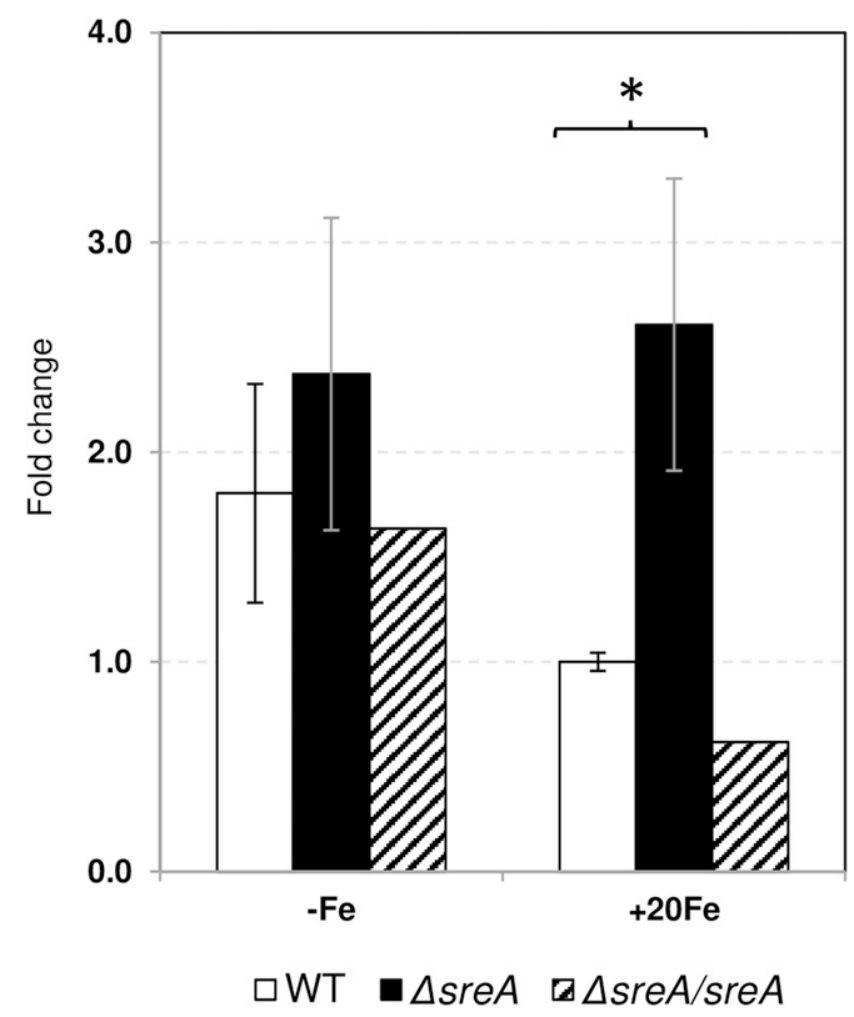

Fig. 3. Effect of Epichloë festucae SreA mutation on fetC expression. Results of relative real-time quantitative PCR used to quantify fetC gene transcripts present in liquid cultures of wild-type (WT) $(n=3), \Delta s r e A(n=$ $4)$, and $\Delta$ sreA/sreA $(n=1)$ strains after 2 days of supplementation with either $100 \mu \mathrm{M}$ bathophenanthroline disulfonic acid or $50 \mu \mathrm{M} \mathrm{FeCl}_{3}(+\mathrm{Fe})$ at $23^{\circ} \mathrm{C}$ (Materials and Methods). Units are given relative to a reference gene $(a c t G)$, and samples were calibrated relative to the WT $(+\mathrm{Fe})$ sample. Error bars are standard deviation. concentration treatments (Supplementary Fig. S8). The $\Delta s r e A-$ infected plants appear to contribute mostly to this delay and the result suggests that the lowest iron concentration alleviates the effect of $\Delta s r e A$ on the rate of chlorosis and is in keeping with SreA requiring iron to function. To assess the effect of endophyte on host growth under the low-iron conditions (above), at day 23, we measured plant heights for a subset of plants. These randomly selected plants consisted of duplicate plants of $9 \mathrm{WT}$ and $10 \Delta s r e A$-infected genotypes for each of the three treatment conditions (i.e., 114 of 180 plants). Over all iron treatments, the mean height of the $\Delta s r e A$-infected plants $(31.5 \pm 1.2 \mathrm{~cm})$ was significantly $(P<0.0001)$ reduced compared with WT-infected plants $(39.5 \pm 0.7 \mathrm{~cm})$, indicating that $\Delta s r e A$ strains can negatively affect host growth. Application of iron $(50 \mu \mathrm{M})$ to chlorotic plants completely restored green coloration $(\mathrm{TC}>$ $25 \mu \mathrm{g} / \mathrm{cm}^{2}$ ) to all plants by 6 days (Supplementary Fig. S9). To evaluate the contribution of endophyte biomass to the phenotypes, we quantified the endophyte gDNA in tiller segments of a subset of WT- and $\Delta s r e A$-infected plants $(n=13$ and 15 plants, respectively) that had been subjected to the $0.25-\mu \mathrm{M}$ iron treatment (Materials and Methods). The number of copies of the NRPS1 gene per nanogram of total gDNA was higher in the $\triangle$ sreA-infected plants than those infected with the WT (endophyte DNA at $185 \pm 112$ and $98 \pm 46$ copies $\mathrm{ng}^{-1}$ of total gDNA, respectively; $P=0.014$, ANOVA). This result indicates generally more $\triangle s r e A$ nuclei than WT present in the $0.25-\mu \mathrm{M}$ samples, which may contribute to overharvesting of iron by $\triangle s r e A$ strains. However, there was no correlation $(P>0.05)$ of endophyte biomass with either plant height or the rate of chlorophyll decline (not shown), although there was a moderate correlation rate of chlorosis and plant height $(R=0.386, P=$ 0.000 , Pearson). This suggests that endophyte biomass might not be a primary driver of iron overconsumption in $\Delta s r e A-$ infected plants. Overall, the results indicated that SreA deficiency leads to overharvesting of host iron which can be detected as leaf chlorosis but only in iron-limiting environments, and suggests that SreA plays a role in moderating iron acquisition within host plants.

\section{DISCUSSION}

In maintaining long-term mutualistic interactions, both symbiotic partners must be capable of contributing certain benefits as well as tolerances during the intimate cohabitation. As dedicated grass-shoot endosymbionts, one of the caveats for the Epichlö spp. during plant vegetative growth is that they must gain nutrients from the host apoplasm without overharvesting provisions which could, in addition to depriving the host of nourishment, result in overcolonization of the intercellular niche and, thus, jeopardize the viability and persistence of the association. This research describes an aspect of E. festucae iron regulation that may have implications for symbiotic stability. The study increases our understanding of factors involved in plant-fungus interactions and the importance of regulation of iron acquisition in maintaining mutualism.

A single gene (sreA) candidate for the E. festucae IRGF ortholog was identified, encoding SreA, that contains all key conserved amino acid motifs described for equivalent proteins from many filamentous fungi (An et al. 1997b; Pelletier et al. 2005). Moreover, functional studies revealed, in similarity to the IRGFs of other fungi, the consequence of SreA mutation for E. festucae, including sensitivity to iron (Fig. 1) and derepression of siderophore biosynthesis (Fig. 2) and iron uptake (Fig. 2, inferred in Figure 3) in the presence of iron. Together, these results indicate conservation of the IRGF repressor function for the genus Epichlö̈ as in other fungal taxa. 
More specifically, the axenic culture growth phenotypes observed under differing iron concentrations are consistent with those associated with the loss of other SreA orthologous proteins from other fungi (Chen et al. 2011; Hwang et al. 2011; Jung et al. 2006; Oberegger et al. 2001; Schrettl et al. 2008). During the course of our studies, we have noted a reduced diameter and altered morphology and pigmentation similar to that observed for $\Delta$ sreA colonies supplemented with $50 \mu \mathrm{M}$ $\mathrm{FeCl}_{3}$ (Fig. 1). These symptoms were also observed for WT colonies but only at significantly higher iron supplementation $(400 \mu \mathrm{M} \mathrm{FeCl})_{3}$ ). In addition, $1 \mathrm{mM} \mathrm{FeCl}{ }_{3}$ supplementation prevented E. festucae growth (not shown) and, therefore, it
A

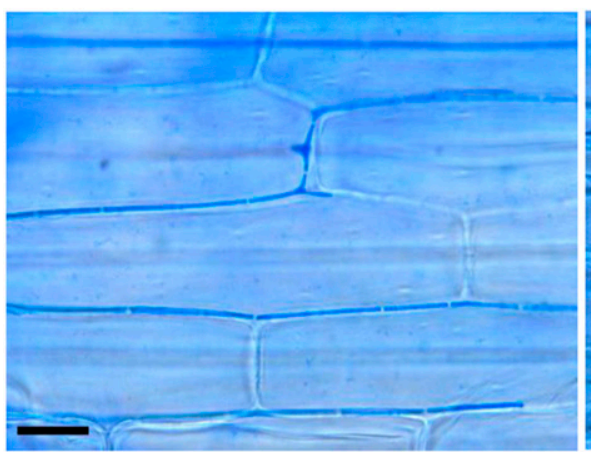

B

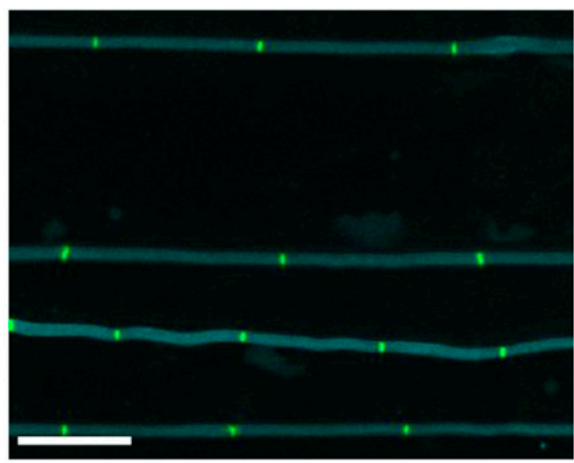

C

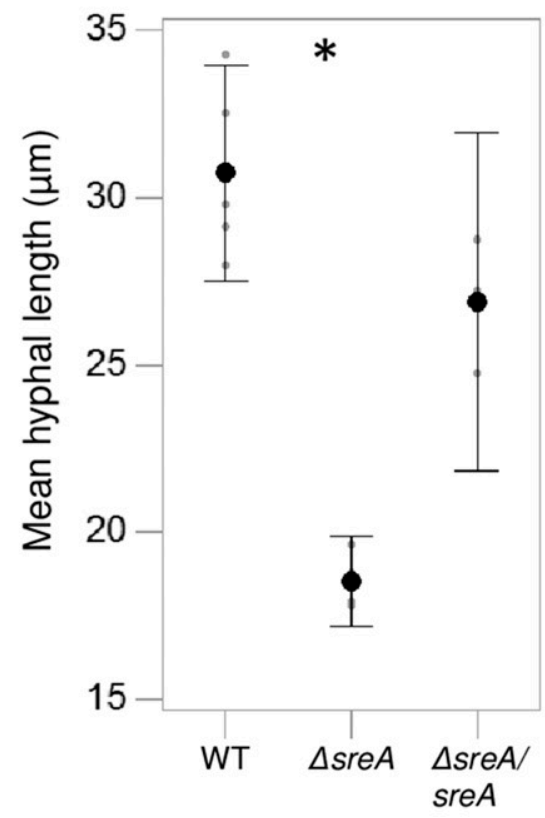

$\Delta s r e A$

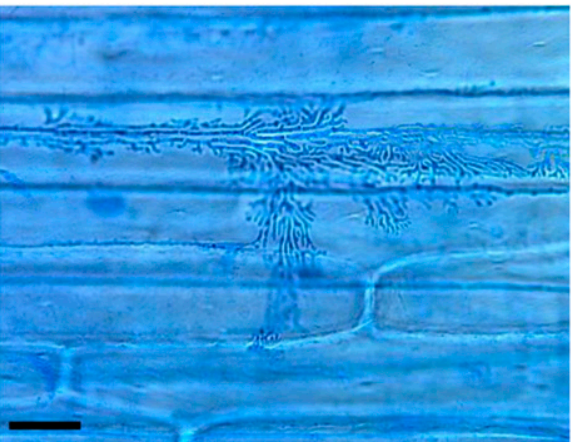

$\Delta s r e A$

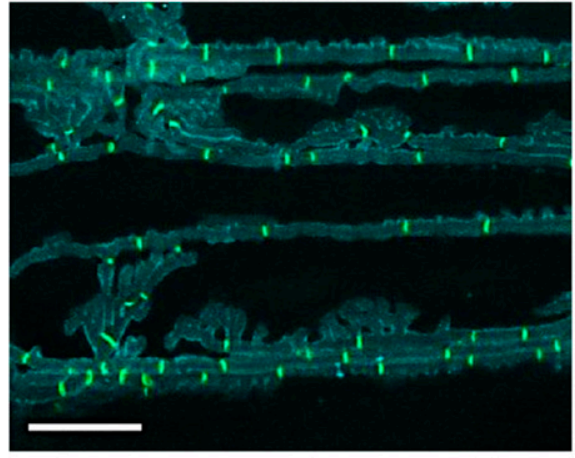

D

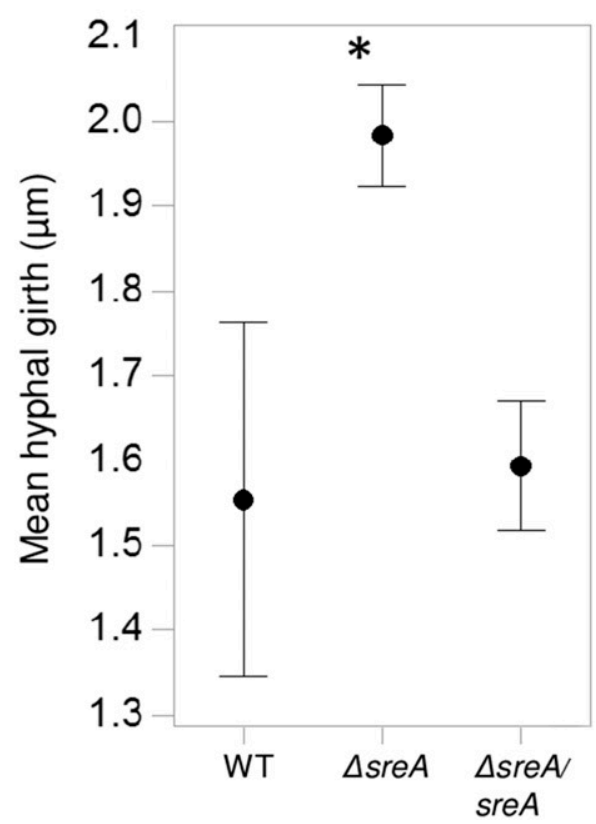

$\Delta$ sreA/sreA

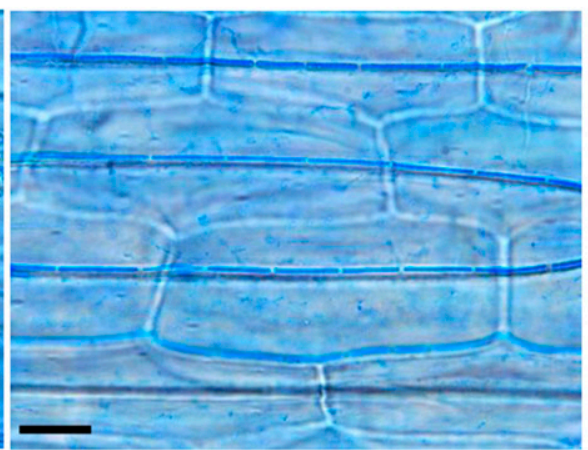

$\Delta$ sreA/sreA

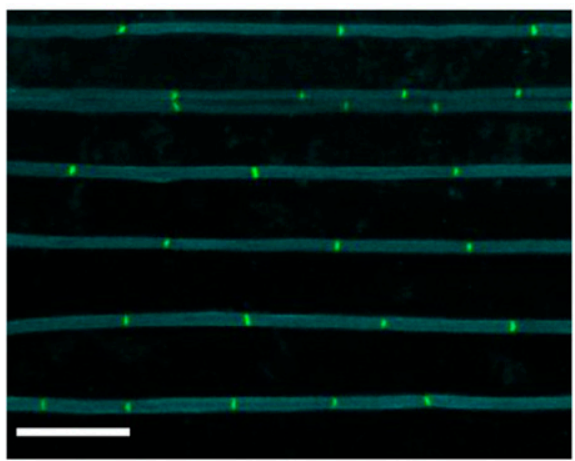

$\mathbf{E}$

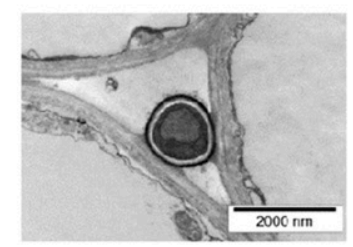

$\triangle$ sreA

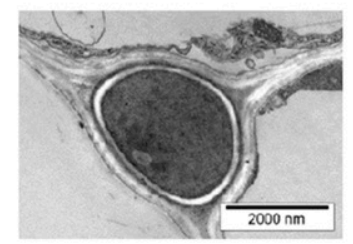

$\triangle \operatorname{sre} A$

sreA

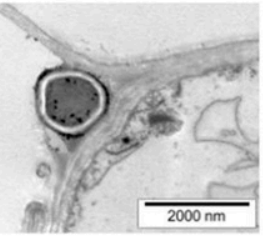

Fig. 4. Growth characteristics of $\Delta s r e A$ in ryegrass plants. Representative A, brightfield and $\mathbf{B}$, confocal microscope image captures of wild-type (WT), $\Delta s r e A$, and $\Delta s r e A /$ sreA hyphae, stained aniline blue and septa highlighted using WGA-488 (green). Their hyphal dimensions (in micrometers) of $\mathbf{C}$, length and $\mathbf{D}$, diameter were obtained using confocal or $\mathbf{E}$, transmission electron microscopy imaging, respectively. Bars in A and B $=20 \mu \mathrm{m}$. Error bars in C and D are standard deviation. An asterisk denotes a significant difference between the mutant and control strains as determined by analysis of variance, $* P<0.05$ (Minitab, v17.21). 
seems likely that the colony phenotype of $\Delta s r e A$ is a response to iron toxicity.

Consistent with studies from other filamentous fungi, derepression of siderophore biosynthesis was observed with $\Delta s r e A$ mutants (Haas et al. 1999; Hwang et al. 2011; Oberegger et al. 2001; Zhou et al. 1998). However, the unexpected elevated levels of FC in iron-deprived $\triangle s r e A$ cultures were likely caused by residual iron present in the $\triangle s r e A$ starter culture grown in complete medium (potato dextrose broth [PDB]). Nevertheless, this higher starting FC concentration did not conceal the impact on siderophore production upon iron addition or removal when compared with siderophore production of the T0 $(\mathrm{DM} 0$, day $=0)$ cultures. These results reiterate our earlier results (Forester et al. 2018), indicating that $\mathrm{FC}$ production is promoted by iron; however, whether the FC accumulation in iron-supplied $\Delta s r e A$ strains is exacerbated by SreA mutation or simply the result of increased iron availability in $\triangle s r e A$ strains is not certain. In contrast, the iron-induced elevation of intracellular FEA (rather than repression, as observed for the controls) is evidently SreA influenced. We have preliminary transcriptomic analyses supporting a theory that some of the genes involved in FEA biosynthesis are targets of SreA (N. T. Forester, unpublished data). The delayed repression of extracellular FEA levels in

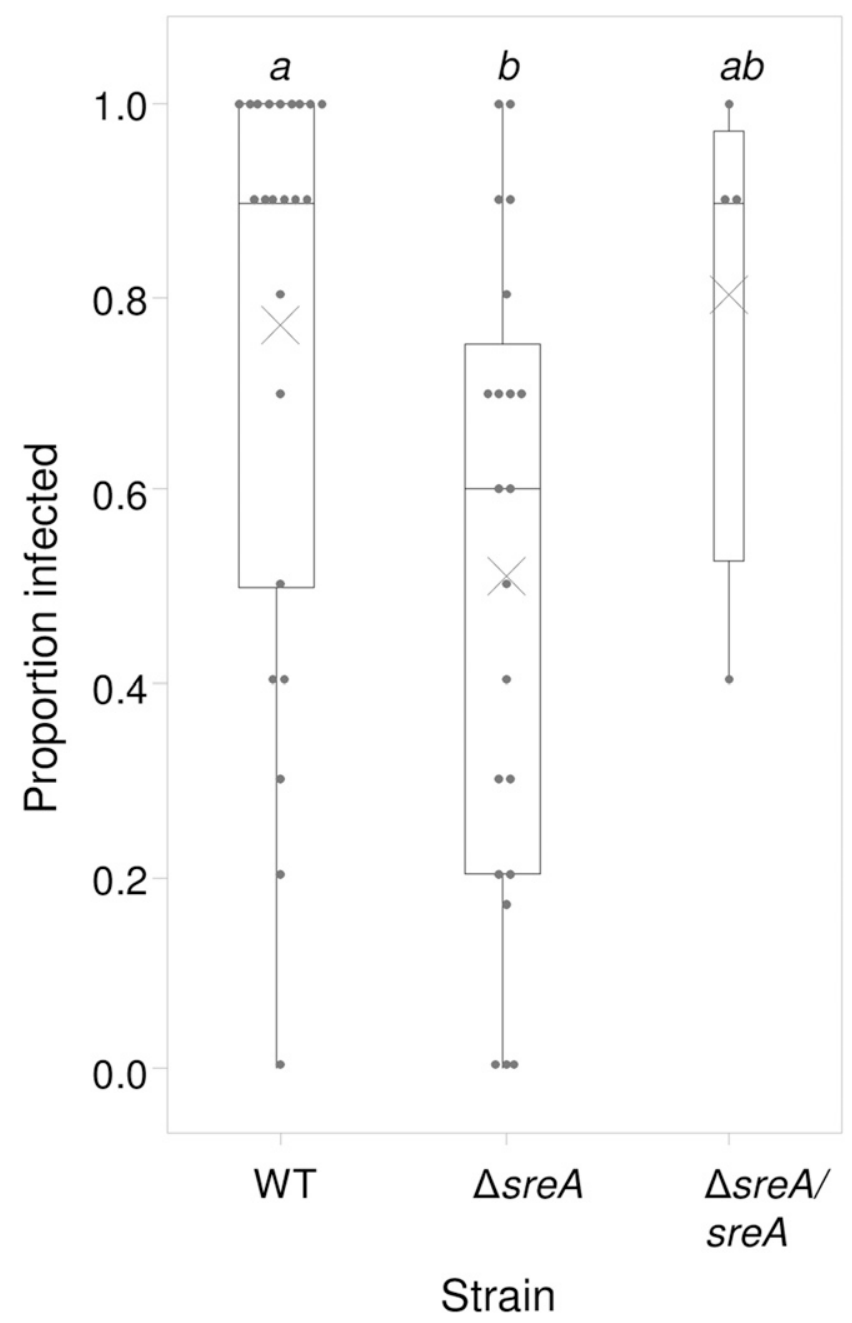

Fig. 5. Persistence of $\Delta s r e A$ in ryegrass plants. Box plot showing the proportion of tillers infected with wild-type (WT), $\Delta s r e A$, or $\Delta s r e A /$ sreA strains in different host genotypes (denoted by dots). Italicized letters denote groups assigned by Tukey's pairwise method at the $95 \%$ confidence level (Minitab, 18.0). The X symbol denotes the mean proportion value for each strain. the $\Delta$ sreA strains in the presence of iron (relative to the WT) (Fig. 2) suggests that high abundance of FEA creates a bottleneck for ferrisiderophore uptake. Higher levels of intracellular FEA also suggest increased intracellular ferration of EA aposiderophores, further indicating substantial deregulation of iron uptake by the other iron-uptake systems such as the RIA system.

Related to this, we observed a significant relative increase in the $\triangle s r e A$ strain of transcripts of the fetC gene, encoding the ferroxidase component of the RIA membrane system, in conditions where fet $C$ would normally be downregulated (i.e., under high iron supply) (Fig. 3). Moreover, preliminary transcriptomic analyses in progress of $\Delta s r e A$-infected ryegrass indicate that fet $C$ and $f t r A$ (encoding the iron permease of RIA) are differentially upregulated in planta (unpublished data). These results suggest increased activity of the RIA system in the $\Delta s r e A$ mutants, and we hypothesize that RIA contributes significantly to the intracellular ferric iron retention of the $\triangle s r e A$ strains. RIA likely donates ferric ions to intracellularly located aposiderophores, which is also in keeping with our previous results, implying that RIA is an important iron-uptake system for E. festucae (Forester et al. 2018; Johnson et al. 2013b). Altogether, these results highlight the crucial role of SreA in controlling iron access via high-affinity iron management systems to Epichlö̈ cells to prevent iron build up and the related iron toxicity effects observed in axenic culture.

\section{In planta hyphal growth.}

Within the Epichlo $\ddot{e}$-grass symbiotum, the $\Delta s r e A$ hyphae exhibited clear signs of unusual malformed growth in the apoplasm, especially in those regions associated with expanded host cell tissue. There were reduced hyphal compartment sizes and the frequent occurrence of repeatedly branched hyphae that formed networks of varying sizes, indicative of abnormal branching (Fig. 4). The creation of these features is possibly mediated via regions of cell membrane weakness of hyphal filaments (as suggested by the presence of FC in $\Delta s r e A$ supernatants) that are more prone to distension under turgor pressure. In fact, the whole structure appeared to be more susceptible to physical damage than typical in planta hyphae, with the common observation of residual outlines of networks and increased aniline-blue-stained debris in $\triangle s r e A$ specimens. Given the similarity of FEA biosynthesis to that of triacetylfusarinine C (TAFC), an extracellular siderophore of A. fumigatus, mevalonate, is a shared precursor of both ergosterol and TAFC (and, by inference, FEA) biosynthesis (Yasmin et al. 2012). Ergosterol is a common constituent of fungal cell membranes. Therefore, it is plausible that diversion of mevalonate toward EA production may have consequences for cell structures reliant on the production of ergosterol, possibly limiting the growth of hyphal compartments. Further measurements of different sterol levels in $\Delta s r e A$ mutants are required to confirm this hypothesis of a direct relationship between siderophores, steroids, and Epichlö̈ cell growth.

In addition, other factors such as the presence of reactive oxygen species and changes in cellular $\mathrm{pH}$ may also contribute to the phenotype. Both of these factors are also known to affect the expression of green fluorescent protein (GFP) (Alnuami et al. 2008; Shaner et al. 2007) and be relevant to the $\Delta s r e A$ mutant because we failed to produce useable $\Delta s r e A$ strains expressing enhanced GFP (eGFP) owing to rapid photo-bleaching of the GFP signal (not shown).

\section{Effect on host and symbiosis.}

Virtually nothing is known about how iron regulation affects symbiotic interactions in E. festucae, yet we do know 
that endophyte growth is deregulated upon deactivating mutations of the genes involved in siderophore biosynthesis, which is sensitive to iron availability (Forester et al. 2018; Johnson et al. 2013b). Therefore, we sought to gain insights into the role of SreA and iron regulation in endophytic symbiosis. Consistent with the hypothesis that $\Delta$ sreA mutants are impaired in the control of iron acquisition, manipulation of iron supply to $\Delta s r e A$-infected hosts using hydroponics revealed that, when iron was limited, SreA deficiency led to premature chlorosis which could be remedied with iron supplementation (Fig. 6). Notably, detecting iron overconsumption by $\Delta s r e A$, could only be achieved at an iron concentration range that was close to limiting for the host plant, whereas plants infected with $\triangle s r e A$ remained green using adequate iron supply ( 20 to $50 \mu \mathrm{M})$, which suggests that endophytes generally do not affect host iron availability and, therefore, plant growth. Incidentally, the similarity of the appearance of these plants grown at adequate iron supply and their counterparts that have been grown in soil suggests that endophytes typically experience an iron-sufficient environment in the apoplasm. It is also interesting to note that plants grown using an iron supply $>200 \mu \mathrm{M}$ show signs of iron toxicity (dark blue-green leaves, stunted growth), and this is mirrored in culture where, at similar iron concentrations in defined medium, E. festucae begins to display an iron distress growth response. Comparing this with other filamentous fungi (e.g., A. fumigatus) that appear to have millimolar tolerances to iron (Schrettl et al. 2008), the Epichlö̈ spp. may have developed a higher sensitivity to iron through coevolution with grasses.

Because the rate of chlorosis and reduced plant height did not correlate with the increased biomass of $\Delta s r e A$, these results imply that the fungal biomass increase is not a driver of the observed phenotypes. However, this does not preclude the possibility of increased fungal growth positioned close to developmentally sensitive tissues such as meristematic zones leading to delayed outgrowth from such tissues (through interference with signals for growth). Additionally, increased
RIA activity may contribute to premature chlorosis through direct overharvesting of iron, thus depriving the host of iron, which we predict would delay intercalary growth of the plant cells.

In axenic culture, the $\Delta s r e A / s r e A$ complemented strains appropriately rescued the biological activity of $\triangle$ sreA (i.e., siderophore production and colony morphology) and complemented the in planta growth, with restored WT-like hyphal morphology and compartment girth and length (Fig. 1). However, over time, the $\Delta s r e A /$ sre $A$ strains were inconsistent in their ability to complement the $\Delta s r e A$ in the hydroponic growth assays. Discrepancies in the diagnostic PCR results for strain identity in the persistence assay (TPIB) suggests that the $\Delta$ sreA/sreA complemented strains were unable to maintain genetic integrity of the complementation. Given that the identities of $100 \%$ of the WT- and $\Delta s r e A$-infected strains tested were as expected, we suspect that this is likely the result of using a fosmid containing $\Delta$ sreA. Notably, fosmids allow full access to long lengths of error-free DNA (up to $40 \mathrm{~kb}$ ) and have been successfully used (Forester et al. 2018; Johnson et al. 2013b). However, fosmids can introduce large regions of duplicated DNA into the genome, thus providing stretches of homology that could recombine together and result in DNA loss. However, we selected this approach due to the presence of multiple tandem repeats close to $\Delta s r e A$ which caused difficulties in PCR amplification of complement-generating DNA.

\section{Nonpersistence of $\Delta s r e A$.}

With regard to the lack of $\Delta s r e A$ persistence in host plants, there were no significant issues with infection establishment but, over a 2-year period, symbiosis persistence dropped as hyphae were gradually lost from host tillers (Fig. 5). We suggest that this phenomenon may be related to the $\Delta s r e A$ growth response to iron. In culture, the presence of iron reduced the colony diameters of $\Delta s r e A$ (Fig. 1) and, in planta, iron also reduced hyphal compartment extension (not shown). Efficient vegetative transmission of endophytes into tillers requires systemic infection of tiller growth units (phytomers) early in
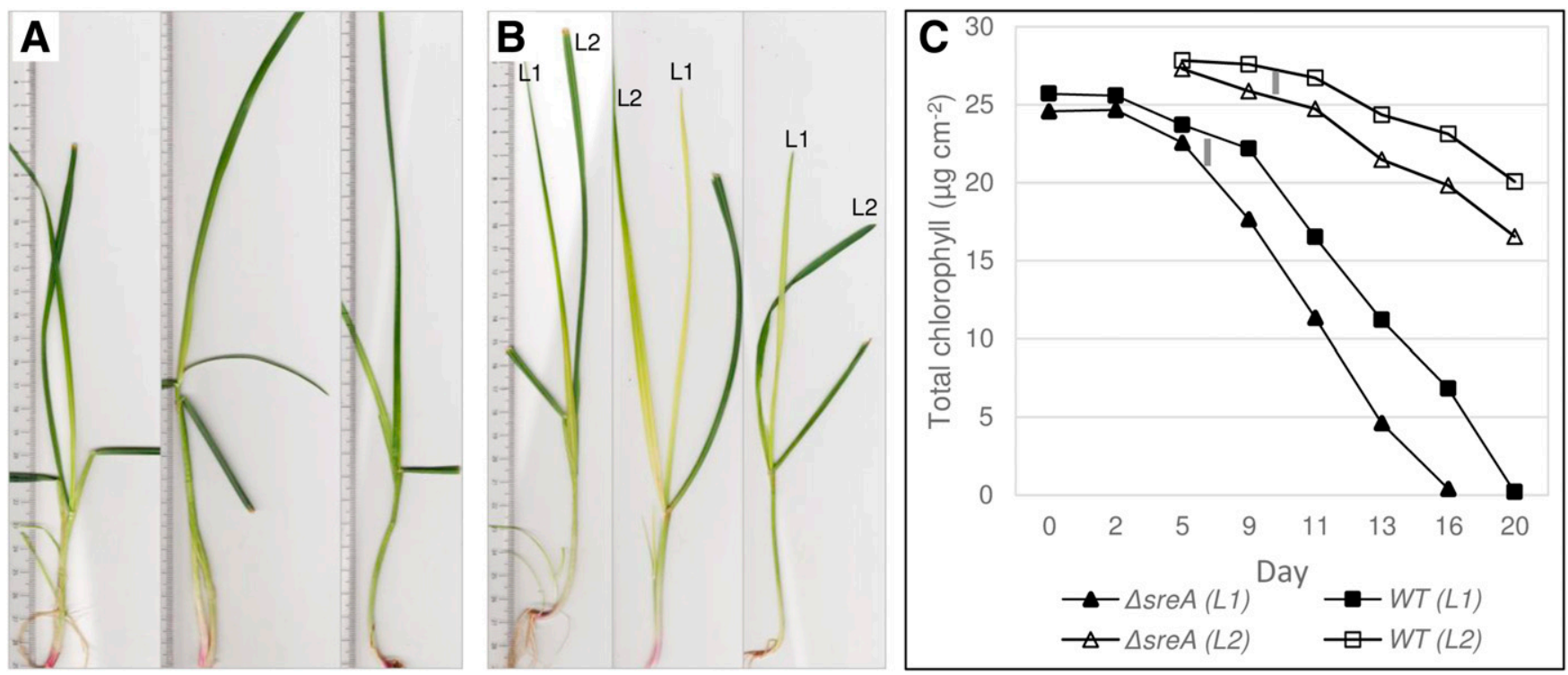

Fig. 6. Effect of Epichloë festucae SreA mutation on host chlorophyll levels over time. Photographs of a single plant tiller hydroponically supplied with A, sufficient iron $(25 \mu \mathrm{M}$ EDTA ferric sodium salt [EDFS] ) or B, low iron $(0.25 \mu \mathrm{M}$ EDFS) indicating the youngest (L1) and second-youngest (L2) expanded leaves measured in the analysis. Each panel shows (from left to right) a representative wild-type (WT)-, $\Delta s r e A-$, or $\Delta s r e A /$ sreA-infected tiller. C, Graph of the mean total chlorophyll (TC) levels (micrograms per square centimeter) in the L1 and L2 leaves of host plants infected with E. festucae Fl1 WT ( $n=85$ ) or $\Delta s r e A(n=89)$. Data values shown represent 15 independent symbioses per strain and the combined average values from all three iron concentrations used $(0.125,0.25$, and $0.5 \mu \mathrm{M}$ of ferric iron as EDFS). Gray vertical bars on graphs represent the average least significant difference for the L1 and L2 comparison (1.4 and $1.5 \mathrm{TC}$ units respectively). 
development. If $\Delta s r e A$ fungi in plants are subjected to sufficient levels of iron, as we suggest, then it is conceivable that the hyphae, with their obvious growth deficiencies and deformities, miss opportunities to infect new tiller primordia. Consequently, new populations of endophyte-free tiller hierarchies would emerge. In agreement with this, we note that $\Delta s r e A$-infected plants grown for a prolonged time without replenishing the potting mix (i.e., nutrient availability becomes reduced over time due to consumption) remain infected. Therefore, SreA plays an important role in maintaining the optimal growth rate in response to iron availability which, in turn, supports longterm in planta persistence. We cannot exclude the possibility, however, that the $\triangle s r e A$ endophyte is lost because it induces a host defense response via recognition of its overproduced siderophores (Dellagi et al. 2009). Additional mechanisms could be the disruption of other host-interaction pathways, or the consequence of deregulation of other gene targets of SreA.

\section{Summary and perspectives.}

It is clear from this work that efficient and appropriate iron management responses (for iron uptake, storage, and utilization) that support E. festucae survival and regulate in planta growth are fundamental to preserve symbiotic interactions during host grass vegetative development. SreA responds to iron and appears to modulate iron accessibility to E. festucae in order to maintain iron homeostasis and prevent iron toxicity. Disruption of the SreA regulator negatively affects the growth relationship of E. festucae with $L$. perenne and may promote resource competition with the host. Therefore, maintenance of iron homeostasis appears important for maintaining a successful mutualistic grass-endophyte association. The E. festucae SreA is expected to have many gene targets and pleiotropic effects, as does its counterparts in A. fumigatus (Schrettl et al. 2008). We are currently conducting transcriptome analyses in an attempt to understand the gene targets of the E. festucae transcription factor and the molecular processes underpinning the changes observed in response to loss of SreA in this interaction.

\section{MATERIALS AND METHODS}

\section{Biological material and growth conditions.}

E. festucae (Leuchtmann et al. 2014) strain Fl1 (originally isolated from ex-cultivar SR3000) that was used in this study was isolated from an infected $L$. perenne plant using a hyphal grow-out method described previously (Clark et al. 1983) and maintained on potato dextrose medium (Oxoid; Sigma), following genetic confirmation of identity using simple-sequence repeat analysis. Lists of all biological materials (microbiological strains and genetic resources) generated or used in this study are given in Supplementary Table S3. Axenic culturing of fungi was performed on potato dextrose or DM, as described previously (Forester et al. 2018). DM0 (+0Fe) is DM without added iron but does contain trace iron (less than $0.6 \mu \mathrm{M}$ ), whereas $-\mathrm{Fe}$ is DM $+100 \mu \mathrm{M}$ BPS (Sigma). BPS is a ferrous iron-chelating agent that was used to induce severe iron deficiency. Growth assays were performed at least three times, with colonies grown for 10 to 14 days at $23^{\circ} \mathrm{C}$ before colony diameter measurements were collected. Representative colonies from one of these assays are shown in the results.

\section{Standard molecular techniques.}

Plasmid or fosmid, fungal gDNA, and total RNA were isolated as previously described (Forester et al. 2018). Likewise, cDNA synthesis, standard PCR, and qRT-PCR were performed as previously described (Forester et al. 2018). A list of all primers used in this study is presented in Supplementary
Table S4. To quantify fetC transcripts, the primers pairs used were LJNTF2:09 and 2:10 for fet $C$ and LJNTF2:27 and 2:28 for the reference gene, E. festucae act $G$. The cDNA template was generated from steady-state liquid cultures of WT (A, 1, and 4), $\Delta$ sreA (1-9, 7-9, and 9-11) and $\Delta$ sreA/sreA (1-8) strains that had been grown for 2 days (at $23^{\circ} \mathrm{C}$ ) in DM0 supplemented with either $100 \mu \mathrm{M}$ BPS or $50 \mu \mathrm{M} \mathrm{FeCl}_{3}(+\mathrm{Fe})$. The starter culture and treatment cultures was prepared as previously described (Forester et al. 2018) but using $50 \mathrm{ml}$ of DM0 in place of PDB to recover the mycelia prior to treatments. qRT-PCR was performed on cDNA templates generated from total RNA preparations of each strain using primers specific for $f e t C$ and act $G$ (above). Reactions were carried out using the LightCycler 480 System (Roche Diagnostics) and SYBR Green I detection according to the manufacturer's instructions. The amplification conditions (ramp rates $4.4^{\circ} \mathrm{C} / \mathrm{s}$, unless stated) were preamplification denaturing step at $95^{\circ} \mathrm{C}$ for $5 \mathrm{~min}$; then, 40 amplification cycles of $95^{\circ} \mathrm{C}$ for $20 \mathrm{~s}, 59^{\circ} \mathrm{C}$ (ramp rate $2.2^{\circ} \mathrm{C} / \mathrm{s}$ ) for $10 \mathrm{~s}$, and $72^{\circ} \mathrm{C}$ for $10 \mathrm{~s}$; followed by a melt curve analysis (continuous acquisition mode, five acquisitions per $1^{\circ} \mathrm{C}, 0.11 \mathrm{ramp}$ rate from $65^{\circ} \mathrm{C}$ ). Data were analyzed using advanced relative quantification experiments, with efficiencies obtained from external standards and referencing a calibrator sample according to the Light Cycler software instructions (Roche). Mean calculated crossing point values for triplicate reactions per sample were obtained using an "all to mean" pairing rule in the LightCycler 480 Software. The mean fold changes were calculated relative to the reference gene and a calibrator treatment $(\mathrm{WT},+\mathrm{Fe})$.

\section{Generation of $E$. festucae transformants.}

Deletion of $\triangle s r e A$ was achieved using a MultiSite Gateway three-fragment Vector Construction Kit (Invitrogen) to make a construct to replace the entire gDNA encoding sreA with a hygromycin B resistance $(h p h)$ gene cassette (Supplementary Fig. S10). Two entry clones, pENTR-5' sreA and pENTR-3' sreA, were each generated using sreA-flanking sequences that were PCR amplified from gDNA of E. festucae strain Fl1. Subsequently, the destination vector pDEST- $\Delta$ sreA was produced in a Gateway LR reaction from the two entry clones and pENTR-Hyg ${ }^{\mathrm{R}}$ was used to transform E. festucae Fl1 protoplasts, as described previously (Forester et al. 2018). Homologous recombinants were generated by polyethylene glycol-mediated protoplast transformation, as described previously (Johnson et al. 2013b), and transformants were selected using hygromycin B $(150 \mu \mathrm{g} / \mathrm{ml}$; Invitrogen). Individual colonies of protoplasts regenerated without DNA or without hygromycin selection were used as WT controls. Of 90 single-nuclear purified transformants, 5 contained the desired deletion event by screening using multiplex PCR. Four selected transformants ( $\triangle$ sreA 1-9, 7-9, 811, and 9-11) were then verified for a single locus insertion by Southern blot analysis using the digoxigenin system (Roche). Blotted membranes were queried twice using probes specific for the respective genes, spanning sreA (primers LJNTF1:50 and $1: 51,527 \mathrm{bp}$ ) or the $h p h$ gene encoding hygromycin resistance (primers LJNTF1:73 and 1:74, 385 bp). For genetic complementation of $\Delta s r e A$, the $\Delta s r e A 1-9$ protoplast cells were cotransformed with fosmid clone 5P23 DNA $(5 \mu \mathrm{g})$, which is an Fl1-derived fosmid containing approximately $35 \mathrm{~kb}$ of genomic sequence, including the full intact sreA locus generated previously (Johnson et al. 2013b), and $1 \mu \mathrm{g}$ of plasmid pII99 (Namiki et al. 2001) which, upon ectopic integration into the $E$. festucae genome, confers resistance to geneticin $(200 \mu \mathrm{g} / \mathrm{ml})$. Two ( $\Delta$ sreA/sreA 5P23-1-8 and 5P23-1-15) of five potential complemented strains that assayed positive for WT-like behavior in axenic culture iron tests and siderophore assays were chosen as control strains. 


\section{L. perenne infection.}

Endophyte-free L. perenne seed (diploid perennial ryegrass: accession A11104; Margot Forde Germplasm Centre) were heat treated to reduce saprophytes and further surface sterilized as previously described (Forester et al. 2018). Seedlings were then inoculated with fungal strains and transferred to soil to grow for 8 to 10 weeks at ambient glasshouse conditions, and the surviving plants were subjected to various examinations, including phenotyping and microscopy, as previously described (Forester et al. 2018). The infection status of each plant was confirmed by the TPIB method of Simpson et al. (2012) and by standard brightfield microscopy (BX50; Olympus) using an aniline blue staining method, as previously described (Tanaka et al. 2005). In planta strain identity was confirmed using standard PCR on gDNA derived from symbiota tissue using multiple primer combinations and comparing banding patterns. Multiplex primer combinations of LJNTF1:44, 1:47, and 3:09 yield for WT and $\triangle s r e A$ a 889- and 394-bp product, respectively, whereas both products are observed for $\Delta$ sreA/sreA. Primers LJNTF4:03 and 4:04 yield a 248-bp vector band that is diagnostic for the complemented strain. Notably, these priming sites are present in both pDEST- $\Delta$ sre $A$ and the fosmid 5P23-1-8 but the version from pDEST- $\Delta$ sre $A$ was not incorporated into the genome as shown in the banding patterns for $\Delta s r e A$. Research with genetically modified organisms comes under approval code GMD002078, in accordance with the New Zealand regulatory requirements.

\section{Measurements of $\boldsymbol{E}$. festucae siderophores.}

Sample preparation, solvent extraction, and quantitative LCMS analysis of siderophores was carried out as described in our previous study (Forester et al. 2018).

\section{Hydroponic plant growth and chlorophyll measurements.}

Hydroponic solution (HS) used in this study was a modified recipe of Hoagland and Arnon (1950), following the recipe of Graziano (Graziano and Lamattina 2007). It was prepared by making the following concentrates to volume with ultrapure water: $1,000 \times$ potassium phosphate buffer (1 M prepared to $\mathrm{pH} 5.8$ from $1 \mathrm{M} \mathrm{KH}_{2} \mathrm{PO}_{4}$ and $1 \mathrm{M} \mathrm{K}_{2} \mathrm{HPO}_{4}$ ), $1,000 \times$ microelements $\left(24 \mathrm{mM}[0.15 \%] \mathrm{H}_{3} \mathrm{BO}_{3}, 1 \mathrm{mM}\right.$ [0.02\%] $\mathrm{MnCl}_{2} \cdot 4 \mathrm{H}_{2} \mathrm{O}, 0.5 \mathrm{mM}[0.015 \%] \mathrm{ZnSO}_{4} \cdot 7 \mathrm{H}_{2} \mathrm{O}$, $0.3 \mathrm{mM}$ [0.008\%] $\mathrm{CuSO}_{4} \cdot 5 \mathrm{H}_{2} \mathrm{O}$, and $0.2 \mathrm{mM} \mathrm{[0.005 \% ]}$ $\left.\mathrm{NaMoO}_{4} \cdot 2 \mathrm{H}_{2} \mathrm{O}\right)$, and $10 \times$ macroelement solution $(30 \mathrm{mM}$ $[0.3 \%] \mathrm{KNO}_{3}, 15 \mathrm{mM}[0.35 \%] \mathrm{Ca}\left(\mathrm{NO}_{3}\right)_{2} \cdot 4 \mathrm{H}_{2} \mathrm{O}$, and $15 \mathrm{mM}$ [0.37\%] $\mathrm{MgSO}_{4} \cdot 5 \mathrm{H}_{2} \mathrm{O}$ ). Chelated EDTA ferric sodium salt (EDFS) (CAS number 15708-41-5; Sigma) was added to the indicated concentrations $(0.2$ to $100 \mu \mathrm{M})$. Individual plant tillers were cut transversely with a scalpel below the third leaf-stem junction and the leaf blade length (measured from the outermost leaf) was reduced by approximately two-thirds. The tillers were surface sterilized by immersing in $10 \%$ "Janola" (commercial bleach in ultrapure water) for $5 \mathrm{~min}$ (room temperature), followed by five 5-min washes in sterile water and air drying. To minimize iron leaching from colored plastic ware, for each hydroponic growth unit, a clear plastic cup $(275 \mathrm{ml})$ was used, with an A4-sized transparency film rolled into a cylinder (with plants attached), fastened, and inserted portrait orientation into the cup containing $100 \mathrm{ml}$ of HS (plus $20 \mu \mathrm{M}$ EDFS). Three prepared tillers were mounted in tabs cut equidistant on the film and the cup-and-pot assembly was inserted into a black sleeve to reduce light to roots. which were regenerated over the next 10 days prior to iron treatments. The solution was changed every 5 days. Hydroponically treated plants were grown in a plant growth room (constant $20^{\circ} \mathrm{C}$, 9-h light photoperiod, high-pressure sodium grow lights). Two adjacent blocks (A and B) of 30 pots in a 6-by-5 array were positioned on a grid (approximately 0.75 by $0.6 \mathrm{~m}$ ) and the orientations of each pot were maintained throughout the experiment. Each growth unit (pot) contained three tillers infected with the same endophyte strain, these three chosen randomly from 15 ryegrass genotypes infected either with WT (A, 1, or 4) or $\Delta$ sreA (1-9, 7-9, or 9-11) endophytes. Per block, every genotype had a tiller growing under each of three separate iron concentrations $(0.125,0.25$, and $0.5 \mu \mathrm{M}$ ferric iron, supplied as EDTA ferric sodium salt). Upon treatment, measurements were taken for at least 20 days as follows. At indicated time points, the chlorophyll levels of the youngest (L1) and second-youngest (L2) expanded leaf were assessed using an arbitrary leaf color measurement (0 to 90$)$, which was later converted to chlorophyll readings from a standard curve generated with an atLEAF chlorophyll meter (atLEAF). A linear mixed-effects model was used to compare differences between strain (WT and $\Delta s r e A)$, iron concentration $(0.125,0.25$, and $0.5 \mu \mathrm{M}$ EDFS), and day $(0,2,5,9,11,13,16$, and 20). Modeling was undertaken using the nlme and predictmeans packages in $\mathrm{R}$ software (R Core Team 2018).

At day 23, the heights of hydroponically grown plants were measured as the length of the third fully extended leaf, and a linear mixed-effects model (REML) was used to compare differences between strain (WT and $\Delta s r e A$ ) and iron concentration $(0.125,0.25$, and $0.5 \mu \mathrm{M}$ EDFS $)$. The data were analyzed using Genstat, 18th edition (VSN International 2015). The biomass of the endophytes, expressed as copies of the NRPS1 gene per nanograms of total gDNA (assuming a genome size of $35 \mathrm{Mb}$ for E. festucae; NCBI assembly accession GCA_000226195.2), was quantified following the method of our colleagues (Rasmussen et al. 2007). This employed qRTPCR analysis using the Roche LightCycler 480 (Roche) and the $2 \times$ KAPA SYBR FAST qPCR system (KAPA Biosystems) to estimate biomass from gDNA derived from plant material. The gDNA template was prepared from two pooled frozen tiller segments containing the same endophyte strain (i.e., derived from the same transformant colony) of the WT or $\Delta s r e A$ and that had experienced the same iron treatment $(0.25 \mu \mathrm{M}$ EDFS $)$ from block $\mathrm{A}$ and $\mathrm{B}$. Tiller segments as measured from the bottom of the outer leaf $(3 \mathrm{~mm}$ in length, one per plant, two plants) were processed using the QuickDNA Plant/Seed Miniprep Kit, (catalog number D6020) followed by DNA Clean \& Concentrator Kit, (catalog number D4033; Zymo Research). DNA concentrations were determined using NanoDrop-1000 (Thermo Fisher Scientific) and Qubit 3.0 fluorometric quantitation (Life Technologies). The fungal biomass of the two strains was compared by ANOVA (Minitab Inc. 2017).

\section{Imaging infected plant tissues.}

To examine in planta hyphae, standard brightfield light microscopy techniques were used (BX50; Olympus) to view tissues stained by aniline blue, as described previously (Tanaka et al. 2005), using a $\times 100$ oil objective and 1.3 numerical aperture. Additionally, leaf sheath tissue $(3$ to $4 \mathrm{~cm}$ lengths from the leaf base) were mounted in $1 \times$ PBS ( $\mathrm{pH} \mathrm{7.4)}$ and hyphae were observed directly using confocal laserscanning microscopy (CLSM) (e.g., for eGFP-labeled material). Alternatively, the tissue was processed and stained as previously described (Becker et al. 2015), with the following modifications. Aniline blue stain used for CLSM contained $0.05 \%$ (wt/vol) aniline blue in $0.02 \%$ Tween 20 in PBS (pH 7.4) and was vacuum infiltrated for $60 \mathrm{~min}$. AlexaFluor 488 conjugated to wheat germ agglutinin (AF $488-W G A)(10 \mu \mathrm{g} / \mathrm{ml}$ in aniline blue stain above) was added ( 2 drops) to the specimen for $5 \mathrm{~min}$ prior to specimen observation. Confocal images 
were captured and hyphal compartment measurements taken using a FluoView FV10i Confocal Laser-Scanning Microscope (Olympus) and associated software (Olympus FluoView, ver3.1b) according to the manufacturer's instructions. Excitation (Ex) and emission (Em) wavelengths for each fluorophore were monitored as follows: for aniline blue, Ex $405 \mathrm{nM}$ and Em 420 to $520 \mathrm{nM}$; for WGA-AlexaFluor 488, Ex $499 \mathrm{nM}$ and Em $520 \mathrm{nM}$; and for eGFP, Ex $457 \mathrm{nM}$ and Em 465 to $565 \mathrm{nM}$. Representative images for each strain are shown as Z-stacked slices (13 to 15), each at a $1-\mu \mathrm{m}$ depth to capture images of all hyphae in the specimen area $(\times 60$ objective, numerical aperture of 1.35 , and without electronic zoom). Transmission electron microscopy was performed on transversely cut (1-mm sections) basal pseudostem tissue (1 to $2 \mathrm{~cm}$ ) at the Manawatu Microscopy Imaging Centre. Tissue was fixed, processed, and imaged as described previously (Johnson et al. 2013b).

\section{ACKNOWLEDGMENTS}

We thank J. Chen of Manawatu Microscopy Imaging Centre, Palmerston North, NZ for transmission electron microscopy support; K. Ozturk and L. Mace for technical assistance; and R. Johnson for critical review of this manuscript.

\section{AUTHOR-RECOMMENDED INTERNET RESOURCE}

E. festucae Fl1 genome project database: http://www.endophyte.uky.edu/

\section{LITERATURE CITED}

Alnuami, A. A., Zeedi, B., Qadri, S. M., and Ashraf, S. S. 2008. Oxyradicalinduced GFP damage and loss of fluorescence. Int. J. Biol. Macromol. 43:182-186.

An, Z., Mei, B., Yuan, W. M., and Leong, S. A. 1997a. The distal GATA sequences of the sid1 promoter of Ustilago maydis mediate iron repression of siderophore production and interact directly with Urbs 1, a GATA family transcription factor. EMBO J. 16:1742-1750.

An, Z., Zhao, Q., McEvoy, J., Yuan, W. M., Markley, J. L., and Leong, S. A. 1997b. The second finger of Urbs1 is required for iron-mediated repression of sid1 in Ustilago maydis. Proc. Natl. Acad. Sci. U.S.A. 94: 5882-5887.

Becker, Y., Eaton, C. J., Brasell, E., May, K. J., Becker, M., Hassing, B., Cartwright, G. M., Reinhold, L., and Scott, B. 2015. The fungal cell-wall integrity MAPK cascade is crucial for hyphal network formation and maintenance of restrictive growth of Epichloë festucae in symbiosis with Lolium perenne. Mol. Plant-Microbe Interact. 28:69-85.

Blankenship, J. D., Spiering, M. J., Wilkinson, H. H., Fannin, F. F., Bush, L. P., and Schardl, C. L. 2001. Production of loline alkaloids by the grass endophyte, Neotyphodium uncinatum, in defined media. Phytochemistry 58:395-401.

Bush, L. P., Wilkinson, H. H., and Schardl, C. L. 1997. Bioprotective alkaloids of grass-fungal endophyte symbioses. Plant Physiol. 114:1-7.

Chen, C., Pande, K., French, S. D., Tuch, B. B., and Noble, S. M. 2011. An iron homeostasis regulatory circuit with reciprocal roles in Candida albicans commensalism and pathogenesis. Cell Host Microbe 10:118-135.

Christensen, M. J., Ball, O. J. P., Bennett, R. J., and Schardl, C. L. 1997. Fungal and host genotype effects on compatibility and vascular colonization by Epichlö̈ festucae. Mycol. Res. 101:493-501.

Christensen, M. J., Bennett, R. J., Ansari, H. A., Koga, H., Johnson, R. D., Bryan, G. T., Simpson, W. R., Koolaard, J. P., Nickless, E. M., and Voisey, C. R. 2008. Epichlö endophytes grow by intercalary hyphal extension in elongating grass leaves. Fungal Genet. Biol. 45:84-93.

Clark, E. M., White, J. F. and Patterson, R. M. 1983. Improved histochemical techniques for the detection of Acremonium coenophialum in tall fescue and methods of in vitro culture of the fungus. J. Microbiol. Methods 1:149-155.

Dellagi, A., Segond, D., Rigault, M., Fagard, M., Simon, C., Saindrenan, P., and Expert, D. 2009. Microbial siderophores exert a subtle role in Arabidopsis during infection by manipulating the immune response and the iron status. Plant Physiol. 150:1687-1696.

Fleetwood, D. J., Scott, B., Lane, G. A., Tanaka, A., and Johnson, R. D. 2007. A complex ergovaline gene cluster in Epichlö̈ endophytes of grasses. Appl. Environ. Microbiol. 73:2571-2579.
Forester, N. T., Lane, G. A., Steringa, M., Lamont, I. L., and Johnson, L. J. 2018. Contrasting roles of fungal siderophores in maintaining iron homeostasis in Epichloë festucae. Fungal Genet. Biol. 111:60-72.

Gauthier, G. M., Sullivan, T. D., Gallardo, S. S., Brandhorst, T. T., Vanden Wymelenberg, A. J., Cuomo, C. A., Suen, G., Currie, C. R., and Klein, B. S. 2010. SREB, a GATA transcription factor that directs disparate fates in Blastomyces dermatitidis including morphogenesis and siderophore biosynthesis. PLoS Pathog. 6:e1000846.

Graziano, M., and Lamattina, L. 2007. Nitric oxide accumulation is required for molecular and physiological responses to iron deficiency in tomato roots. Plant J. 52:949-960.

Haas, H. 2003. Molecular genetics of fungal siderophore biosynthesis and uptake: The role of siderophores in iron uptake and storage. Appl Microbiol. Biotechnol. 62:316-330.

Haas, H., Angermayr, K., and Stöffler, G. 1997. Molecular analysis of a Penicillium chrysogenum GATA factor encoding gene (sreP) exhibiting significant homology to the Ustilago maydis urbs 1 gene. Gene 184:33-37.

Haas, H., Eisendle, M., and Turgeon, B. G. 2008. Siderophores in fungal physiology and virulence. Annu. Rev. Phytopathol. 46:149-187.

Haas, H., Zadra, I., Stöffler, G., and Angermayr, K. 1999. The Aspergillus nidulans GATA factor SREA is involved in regulation of siderophore biosynthesis and control of iron uptake. J. Biol. Chem. 274:4613-4619.

Harrison, K. A., and Marzluf, G. A. 2002. Characterization of DNA binding and the cysteine rich region of SRE, a GATA factor in Neurospora crassa involved in siderophore synthesis. Biochemistry 41:15288-15295.

Hinton, D. M., and Bacon, C. W. 1985. The distribution and ultrastructure of the endophyte toxic tall fescue. Can. J. Bot. 63:36-42.

Hoagland, D., and Arnon, D. 1950. The Water-Culture Method for Growing Plants Without Soil. College of Agriculture, University of California, Berkeley, CA, U.S.A.

Hwang, L. H., Seth, E., Gilmore, S. A., and Sil, A. 2011. SRE1 regulates iron-dependent and -independent pathways in the fungal pathogen Histoplasma capsulatum. Eukaryot. Cell 11:16-25.

Johnson, L. 2008. Iron and siderophores in fungal-host interactions. Mycol. Res. 112:170-183.

Johnson, L. J., De Bonth, A. C. M., Briggs, L. R., Caradus, J. R., Finch, S. C., Fleetwood, D. J., Fletcher, L. R., Hume, D. E., Johnson, R. D. Popay, A. J., Tapper, B. A., Simpson, W. R., Voisey, C. R., and Card, S. D. 2013a. The exploitation of Epichlö endophytes for agricultural benefit. Fungal Divers. 60:171-188.

Johnson, L. J., Koulman, A., Christensen, M., Lane, G. A., Fraser, K., Forester, N., Johnson, R. D., Bryan, G. T., and Rasmussen, S. 2013b. An extracellular siderophore is required to maintain the mutualistic interaction of Epichloë festucae with Lolium perenne. PLoS Pathog. 9: e1003332.

Jung, W. H., Sham, A., White, R., and Kronstad, J. W. 2006. Iron regulation of the major virulence factors in the AIDS-associated pathogen Cryptococcus neoformans. PLoS Biol. 4:e410.

Kim, H. J., Lee, K. L., Kim, K. D., and Roe, J. H. 2016. The iron uptake repressor Fep1 in the fission yeast binds Fe-S cluster through conserved cysteines. Biochem. Biophys. Res. Commun. 478:187-192.

Leuchtmann, A., Bacon, C. W., Schardl, C. L., White, J. F., Jr., and Tadych, M. 2014. Nomenclatural realignment of Neotyphodium species with genus Epichlö̈. Mycologia 106:202-215.

Li, L., Chen, O. S., McVey Ward, D., and Kaplan, J. 2001. CCC1 is a transporter that mediates vacuolar iron storage in yeast. J. Biol. Chem. 276:29515-29519.

Majewska-Sawka, A., and Nakashima, H. 2004. Endophyte transmission via seeds of Lolium perenne L.: Immunodetection of fungal antigens. Fungal Genet. Biol. 41:534-541.

Mercier, A., and Labbé, S. 2010. Iron-dependent remodeling of fungal metabolic pathways associated with ferrichrome biosynthesis. Appl. Environ. Microbiol. 76:3806-3817.

Miele, R., Barra, D., and Bonaccorsi di Patti, M. C. 2007. A GATA-type transcription factor regulates expression of the high-affinity iron uptake system in the methylotrophic yeast Pichia pastoris. Arch. Biochem. Biophys. 465:172-179.

Minitab Inc. 2017. Minitab 17 Statistical Software. Minitab, Inc., State College, PA, U.S.A.

Namiki, F., Matsunaga, M., Okuda, M., Inoue, I., Nishi, K., Fujita, Y., and Tsuge, T. 2001. Mutation of an arginine biosynthesis gene causes reduced pathogenicity in Fusarium oxysporum $\mathrm{f}$. sp. melonis. Mol. PlantMicrobe Interact. 14:580-584.

Neilands, J. B. 1993. Siderophores. Arch. Biochem. Biophys. 302:1-3.

Oberegger, H., Schoeser, M., Zadra, I., Abt, B., and Haas, H. 2001. SREA is involved in regulation of siderophore biosynthesis, utilization and uptake in Aspergillus nidulans. Mol. Microbiol. 41:1077-1089. 
Oberegger, H., Zadra, I., Schoeser, M., Abt, B., Parson, W., and Haas, H. 2002. Identification of members of the Aspergillus nidulans SREA regulon: Genes involved in siderophore biosynthesis and utilization. Biochem. Soc. Trans. 30:781-783.

Pelletier, B., Beaudoin, J., Mukai, Y., and Labbé, S. 2002. Fep1, an iron sensor regulating iron transporter gene expression in Schizosaccharomyces pombe. J. Biol. Chem. 277:22950-22958.

Pelletier, B., Trott, A., Morano, K. A., and Labbé, S. 2005. Functional characterization of the iron-regulatory transcription factor Fep1 from Schizosaccharomyces pombe. J. Biol. Chem. 280:25146-25161.

Pouliot, B., Jbel, M., Mercier, A., and Labbé, S. 2010. abc $3^{+}$encodes an iron-regulated vacuolar ABC-type transporter in Schizosaccharomyces pombe. Eukaryot. Cell 9:59-73.

Raguzzi, F., Lesuisse, E., and Crichton, R. R. 1988. Iron storage in Saccharomyces cerevisiae. FEBS Lett. 231:253-258.

Rasmussen, S., Parsons, A. J., Bassett, S., Christensen, M. J., Hume, D. E., Johnson, L. J., Johnson, R. D., Simpson, W. R., Stacke, C., Voisey, C. R., Xue, H., and Newman, J. A. 2007. High nitrogen supply and carbohydrate content reduce fungal endophyte and alkaloid concentration in Lolium perenne. New Phytol. 173:787-797.

R Core Team. 2018. R: A Language and Environment for Statistical Computing. R Foundation for Statistical Computing, Vienna, Austria.

Schardl, C. L. 1996. Epichlö̈ species: Fungal symbionts of grasses. Annu. Rev. Phytopathol. 34:109-130.

Schardl, C. L., Leuchtmann, A., and Spiering, M. J. 2004. Symbioses of grasses with seedborne fungal endophytes. Annu. Rev. Plant Biol. 55:315-340.

Schardl, C. L., Young, C. A., Hesse, U., Amyotte, S. G., Andreeva, K., Calie, P. J., Fleetwood, D. J., Haws, D. C., Moore, N., Oeser, B., Panaccione, D. G., Schweri, K. K., Voisey, C. R., Farman, M. L., Jaromczyk, J. W., Roe, B. A., O’Sullivan, D. M., Scott, B., Tudzynski, P., An, Z., Arnaoudova, E. G., Bullock, C. T., Charlton, N. D., Chen, L., Cox, M., Dinkins, R. D., Florea, S., Glenn, A. E., Gordon, A., Güldener, U., Harris, D. R., Hollin, W., Jaromczyk, J., Johnson, R. D., Khan, A. K., Leistner, E., Leuchtmann, A., Li, C., Liu, J., Liu, J., Liu, M., Mace, W., Machado, C., Nagabhyru, P., Pan, J., Schmid, J., Sugawara, K., Steiner, U., Takach, J. E., Tanaka, E., Webb, J. S., Wilson, E. V., Wiseman, J. L., Yoshida, R., and Zeng, Z. 2013. Plant-symbiotic fungi as chemical engineers: Multi-genome analysis of the Clavicipitaceae reveals dynamics of alkaloid loci. PLoS Genet. 9:e1003323.

Schrettl, M., Kim, H. S., Eisendle, M., Kragl, C., Nierman, W. C., Heinekamp, T., Werner, E. R., Jacobsen, I., Illmer, P., Yi, H., Brakhage,
A. A., and Haas, H. 2008. SreA-mediated iron regulation in Aspergillus fumigatus. Mol. Microbiol. 70:27-43.

Shaner, N. C., Patterson, G. H., and Davidson, M. W. 2007. Advances in fluorescent protein technology. J. Cell Sci. 120:4247-4260.

Simpson, W. R., Schmid, J., Singh, J., Faville, M. J., and Johnson, R. D. 2012. A morphological change in the fungal symbiont Neotyphodium lolii induces dwarfing in its host plant Lolium perenne. Fungal Biol. 116: 234-240.

Spiering, M. J., Moon, C. D., Wilkinson, H. H., and Schardl, C. L. 2005. Gene clusters for insecticidal loline alkaloids in the grass-endophytic fungus Neotyphodium uncinatum. Genetics 169:1403-1414.

Stearman, R., Yuan, D. S., Yamaguchi-Iwai, Y., Klausner, R. D., and Dancis, A. 1996. A permease-oxidase complex involved in high-affinity iron uptake in yeast. Science 271:1552-1557.

Tanaka, A., Tapper, B. A., Popay, A., Parker, E. J., and Scott, B. 2005. A symbiosis expressed non-ribosomal peptide synthetase from a mutualistic fungal endophyte of perennial ryegrass confers protection to the symbiotum from insect herbivory. Mol. Microbiol. 57:1036-1050.

VSN International. 2015. GenStat for Windows, 18th ed. VSN International, Hemel Hempstead, U.K.

Voisard, C., Wang, J., McEvoy, J. L., Xu, P., and Leong, S. A. 1993. urbs1, a gene regulating siderophore biosynthesis in Ustilago maydis, encodes a protein similar to the erythroid transcription factor GATA-1. Mol. Cell. Biol. 13:7091-7100.

White, J. F., Jr., Glenn, A. E., and Chandler, K. F. 1993. Endophyte-host associations in grasses. XVIII. Moisture relations and insect herbivory of the emergent stromal leaf of Epichloe. Mycologia 85: 195-202.

Yasmin, S., Alcazar-Fuoli, L., Gründlinger, M., Puempel, T., Cairns, T., Blatzer, M., Lopez, J. F., Grimalt, J. O., Bignell, E., and Haas, H. 2012. Mevalonate governs interdependency of ergosterol and siderophore biosyntheses in the fungal pathogen Aspergillus fumigatus. Proc. Natl. Acad. Sci. U.S.A. 109:E497-E504.

Young, C. A., Felitti, S., Shields, K., Spangenberg, G., Johnson, R. D., Bryan, G. T., Saikia, S., and Scott, B. 2006. A complex gene cluster for indole-diterpene biosynthesis in the grass endophyte Neotyphodium lolii. Fungal Genet. Biol. 43:679-693.

Zhou, L. W., Haas, H., and Marzluf, G. A. 1998. Isolation and characterization of a new gene, sre, which encodes a GATA-type regulatory protein that controls iron transport in Neurospora crassa. Mol Gen. Genet. 259:532-540. 